\title{
Lung Ultrasound in Critically Ill Patients Comparison with Bedside Chest Radiography
}

\author{
Doaa Elsaied Ahmed ${ }^{*}$, Ragia Hassan Hashem ${ }^{1}$, Soad Sayed Abd Elaal Elgaby ${ }^{1}$, Eman Sobh ${ }^{2}$, Gehan \\ Abd Elrhman Eldesoky ${ }^{1}$ \\ ${ }^{1}$ Anesthesiology \& Intensive Care Department, Faculty of Medicine, Al-Azhar University, Cairo, Egypt \\ ${ }^{2}$ Chest Diseases Department, Faculty of Medicine for Girls, Al-Azhar University, Cairo, Egypt \\ * Correspondence: doaashams8@gmail.com
}

Article history: Received: 16-11-2021

Revised:09-12-2021

Accepted: 20-12-2021

\begin{abstract}
Lung radiology is regularly done for the patients in intensive care units (ICU). Chest x-ray (CXR) consider One of the most easily imaging modalities. However, CXR may be not comfortable for critical patients as well as produce hazards of irradiation. The highly variability of quality of the CXR image make CXR interpretation difficult. To show the effect of lung ultrasound (LUS) in ICU patients and diagnose pulmonary diseases compare with chest radiography. This was a prospective study done on 60 patients has acute dyspnea transmitted to the ICU of Alzahraa University Hospital. Sonographic examination of the chest and chest radiographs done to all patients. Sixty patients included in the study with acute dyspnea, CXR and LUS was done to all the patients and then compare between two modalities for diagnosis of pulmonary diseases. Ultrasound is abed side tool useful for rapid and early diagnosis of acute dyspnea in critical ill patients. LUS represents high sensitivity and high specificity of diagnosis of chest diseases as pneumonia, pleural effusion, pneumothorax and interstitial lung diseases compared to CXR.
\end{abstract}

Keywords: Lung ultrasound; Chest X-ray; ICU patients.

\section{INTRODUCTION}

Lung imaging performed for patients in ICU.CXR has limited diagnostic performance and efficacy due to poor quality $\mathrm{x}$ ray films, expose the patients to radiation and need film cassette to placed posteriorly to the thorax. ${ }^{1}$

Another diagnostic tool used frequently is CT chest. However, transport of critical patients to department of radiology increased risk of morbidity and mortality. So using bedside LUS for examination of the lungs is gaining popularity among ICU. ${ }^{2}$

Ultrasound machines that used in ICU should be portable, light- weight and able to store images and videos. LUS display lung images that are depends on both real images and artifacts. LUS can be easily detected pathological conditions that affect the aeration of the lungs and other pleural diseases. ${ }^{3}$

The aim of the study is to show the function of lung ultrasound in ICU patients and diagnose pulmonary diseases comparison with chest radiography.

\section{METHODS}

\subsection{Sample size calculation:}

The sampling technique convenience sampling method. patients were recruited throughout the study period. The sample size was calculated using the clinical calculator. ${ }^{4}$ The minimum sample size required was 49 using the sensitivity and specificity from previously published studies.

\subsection{Study population}

This prospective study involved 60 patients admitted to the ICU of Alzahraa University Hospital suffering from acute dyspnea. All the patients had lung ultrasound, and chest radiographs.

Ethical issues: the study protocol was approved by the Institutional Review Board of Faculty of Medicine for Girls (AFMG-IRB) before patient enrollment in the study. Informed written consents obtained from patients themselves or from their relatives.

\subsection{Statistical Analysis}

- Data are collected, coded on an excel sheet (Microsoft Excel 2010), and statistically analyzed by Statistical Package for Social Science (SPSS) version 19 (SPSS Inc, Chicago, USA). We used Unpaired Student T-test or Mann-Whitney test as appropriate.

- Before the start of the study, we evaluated intra-operator and inter-operator variability and reliability of the readers: Mean and SD were calculated for each reading, and for variation among multiple readings, paired-samples T-test was used to calculate the difference between readers. The intra-

Cite this article: Ahmed, D., Hashem, R., Elgaby,S., Sobh, E. \& Eldesoky, G. Lung Ultrasound in Critically Ill Patients Comparison with Bedside Chest Radiography. Azhar International Journal of Pharmaceutical and Medical Sciences, 2021; 1 (3):118-124. doi: 10.21608/aijpms.2021.106347.1094 
class correlation coefficients (ICC) were calculated for the assessment of intra- and inter-rate reproducibility between learner and expert. The ICC showed good to excellent agreement between readers.

- Sensitivity, specificity, positive predictive value, negative predictive values, and accuracy were measured for lung US results compared to chest CXR results. The gold standard was the final diagnosis obtained by all available tools.

\subsection{Methodology:}

Proper medical history was obtained. We recorded age, sex, results of lab investigations, respiratory support, results of ECG, and echocardiography if indicated.

\subsection{Imaging.}

- $\quad$ Chest X ray was performed immediately after admission and reviewed by one of the investigators.

- Lung ultrasound examination was performed no more than $6 \mathrm{~h}$ of admission. We used (Sonosite M-turbo, FUGIFILM, inc, Usturalia) device and low frequency and high frequency probes. All scanning were performed in supine position. The technique used is as following:

- We followed BLUE protocol as approach to reach diagnosis. This involves rapid screening for anterior (two points), lateral (one point) and posterior (one point) chest on both sides. Scanning was performed with low frequency probe and the high frequency probe as appropriate. This allows diagnosis of pneumothorax, pulmonary edema, pulmonary embolism and pneumonia (19). Pneumothorax was diagnosed based on absent lung sliding, multiple A-lines or Stratosphere sign and presence of lung point if partial pneumothorax. consolidation in most cases is based on presence of shred sign or tissue like sign and dynamic air bronchograms, however atelectasis shows tissue sign but with static air bronchograms and lung pulse. Diagnosis of pulmonary edema depends on presence of multiple B-lines (lung rockets) with preserved lung sliding. Diagnosis of pulmonary embolism required the presence of intact lung sliding anteriorly with normal A-lines and peripheral veins thrombosis (20).

\section{RESULTS}

This study included 60 patients with acute dyspnea were admitted to ICU (40 men and 20 women) and their mean age were 58.6.
Table (1): Mode of oxygenation

\begin{tabular}{lc}
\hline \multicolumn{1}{c}{ Character } & No. $(\%)$ \\
\hline Simple face mask & $32(53.33 \%)$ \\
\hline Venturi mask & $11(18.33 \%)$ \\
\hline Non-invasive CPAP & $4(6.67 \%)$ \\
\hline Mechanical Ventilation & $13(21.66 \%)$ \\
\hline \multicolumn{2}{l}{ CPAP. continuous positive air way pressure }
\end{tabular}

Table (2): Chest radiography of the studied population:

\begin{tabular}{c|c|c|}
\hline \multicolumn{2}{c|}{ Character } & No. (\%) \\
\hline \multicolumn{2}{c|}{ Normal } & $6(10.00 \%)$ \\
\hline \multicolumn{2}{c}{ Abnormal } & \multicolumn{2}{|c}{$54(90 \%)$} \\
\hline \multirow{2}{*}{$\begin{array}{c}\text { Opacity type } \\
\text { Costophrenic } \\
\text { angle }\end{array}$} & Homogeneous & \multicolumn{2}{|c}{$36(60 \%)$} \\
\cline { 2 - 3 } & Heterogenous & \multicolumn{2}{|c}{$12(20 \%)$} \\
\cline { 2 - 4 } & Normal & \multicolumn{2}{|c}{$45(75 \%)$} \\
\cline { 2 - 3 } & & \multicolumn{2}{|c}{$15(25 \%)$} \\
\hline
\end{tabular}

Table (3): CXR diagnosis

\begin{tabular}{lc}
\hline \multicolumn{1}{c}{ CXR Diagnosis } & No. $(\%)$ \\
\hline Normal & $6(10.0 \%)$ \\
\hline Hyperinflated lung & $6(10.00 \%)$ \\
\hline COPD & $1(1.67 \%)$ \\
\hline Consolidation & $25(41.67 \%)$ \\
\hline pleural effusion & $15(25.00 \%)$ \\
\hline Pleural effusion and consolidation & $1(1.67 \%)$ \\
\hline Pneumothorax & $4(6.67 \%)$ \\
\hline Pulmonary edema & $2(3.33 \%)$ \\
\hline Total & $60(100.00 \%)$ \\
\hline
\end{tabular}

Table (4): Pathological finding seen by US

\begin{tabular}{llc}
\hline \multicolumn{1}{c}{ Abnormality } & & No. $(\%)$ \\
\hline Lung slidng & Present & $56(93.33 \%)$ \\
\hline \multirow{2}{*}{ B lines } & Focal B line & $3(5.00 \%)$ \\
\cline { 2 - 3 } & Diffuse B line & $10(16.62 \%)$ \\
\hline A lines & Normal & $58(96.67 \%)$ \\
\hline Lung point & Present & $4(6.67 \%)$ \\
\hline Interrupted Pleural line & Present & $5(8.33 \%)$ \\
\hline Consolidation & Present & $24(40.00 \%)$ \\
\hline \multirow{2}{*}{ Consolidation site } & RT PLAPS & $13(54.17 \%)$ \\
\cline { 2 - 3 } & LT PLAPS & $6(25.00 \%)$ \\
\cline { 2 - 3 } & Bilateral PLAPS & $5(20.83 \%)$ \\
\hline Airbronchogram & Present & $5(8.33 \%)$ \\
\hline Pleural effusion & Present & $20(33.33 \%)$ \\
\hline $\begin{array}{l}\text { Alveolointerstitial } \\
\text { syndrome }\end{array}$ & Present & $5(8.33 \%)$ \\
\hline
\end{tabular}


Table (5): US diagnosis

\begin{tabular}{lc}
\hline \multicolumn{1}{c}{ Diagnosis by US } & No. $(\boldsymbol{\%})$ \\
\hline Pneumonia & $13(21.67 \%)$ \\
\hline pleural effusion & $13(21.67 \%)$ \\
\hline Pulmonary edema (cardiogenic) & $3(5.00 \%)$ \\
\hline Acute respiratory distress syndrome (ARDS) & $5(8.33 \%)$ \\
\hline Pneumonia with parapneumonic effusion & $7(11.67 \%)$ \\
\hline Pneumothorax & $4(6.67 \%)$ \\
\hline Asthma, COPD or Status asthmatics (according to BLUE protocol by exclustion) & $14(23.34 \%)$ \\
\hline Pulmonary edema and pneumonia & $1(1.67 \%)$ \\
\hline
\end{tabular}

Table (6): Comparison between US and CXR in sensitivity, Specificity \& accuracy

\begin{tabular}{|c|c|c|c|c|c|c|c|c|}
\hline Disease & $\begin{array}{c}\text { Radiology } \\
\%\end{array}$ & $\begin{array}{c}\text { Sensitivity } \\
\%\end{array}$ & $\begin{array}{c}\text { Specificity } \\
\%\end{array}$ & $\begin{array}{c}\text { PPV } \\
\%\end{array}$ & $\begin{array}{c}\text { NPV } \\
\%\end{array}$ & LR+ & LR- & Accuracy \\
\hline \multirow{2}{*}{$\begin{array}{c}\text { Pleural } \\
\text { Effusion }\end{array}$} & US & $94 \%$ & $96 \%$ & $98.4 \%$ & $91.9 \%$ & 32,9 & 0,047 & $96 \%$ \\
\hline & CXR & $70 \%$ & $90 \%$ & $93.8 \%$ & $62.7 \%$ & 8.27 & 0.32 & $78 \%$ \\
\hline \multirow{2}{*}{ Pneumothorax } & US & $100 \%$ & $100 \%$ & $96.5 \%$ & $94.3 \%$ & 58.3 & 0.063 & $95 \%$ \\
\hline & CXR & $70 \%$ & $81.8 \%$ & $94.4 \%$ & $81.7 \%$ & 35.4 & 0.476 & $84 \%$ \\
\hline \multirow{2}{*}{ Pneumonia } & US & $93 \%$ & $97 \%$ & $98 \%$ & $87.8 \%$ & 38.8 & 0.11 & $93 \%$ \\
\hline & CXR & $70 \%$ & $95 \%$ & $89.4 \%$ & $64.5 \%$ & 6.67 & 0.43 & $74 \%$ \\
\hline \multirow{2}{*}{$\begin{array}{c}\text { Pulmonary } \\
\text { Edema }\end{array}$} & US & $93 \%$ & $93 \%$ & $88.9 \%$ & $98.9 \%$ & 80.81 & 0.112 & $98 \%$ \\
\hline & CXR & $36 \%$ & $90 \%$ & $75 \%$ & $93.7 \%$ & 30.27 & 0.674 & $93 \%$ \\
\hline
\end{tabular}

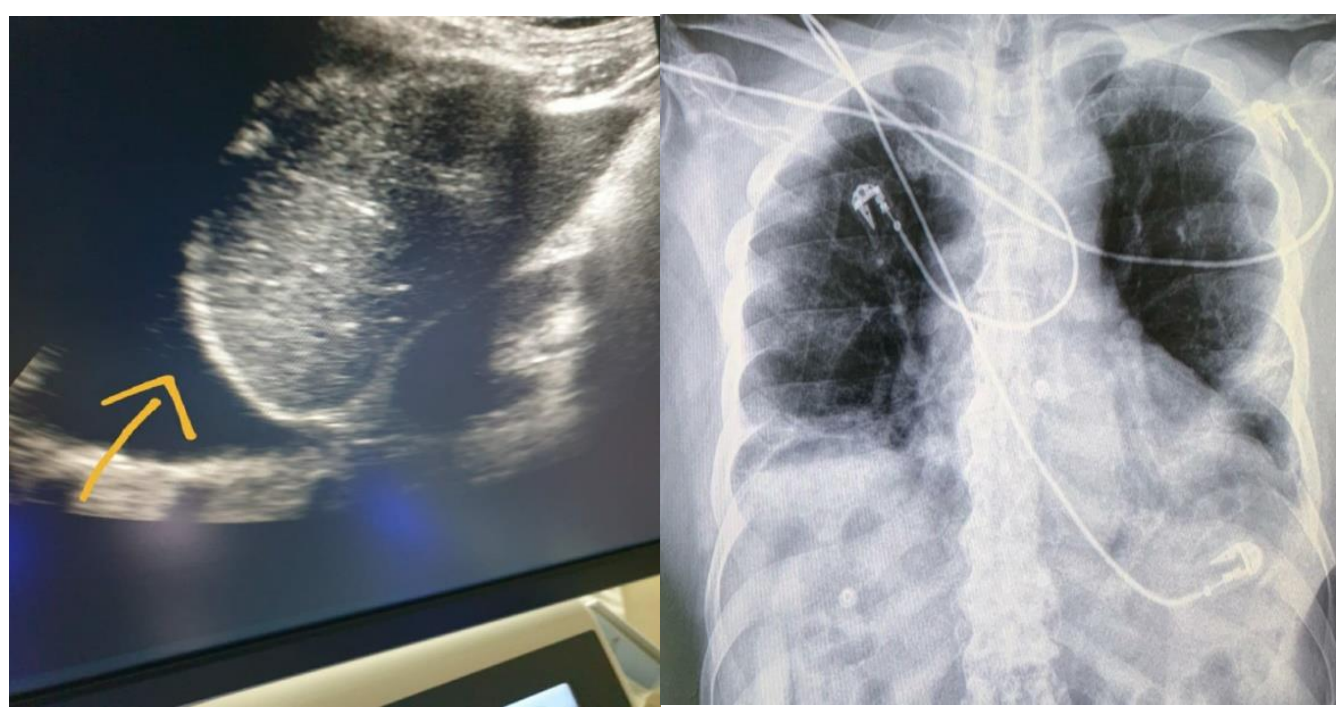

Figure (1 ): picture of pleural effusion in studied patients by LUS and CXR. 


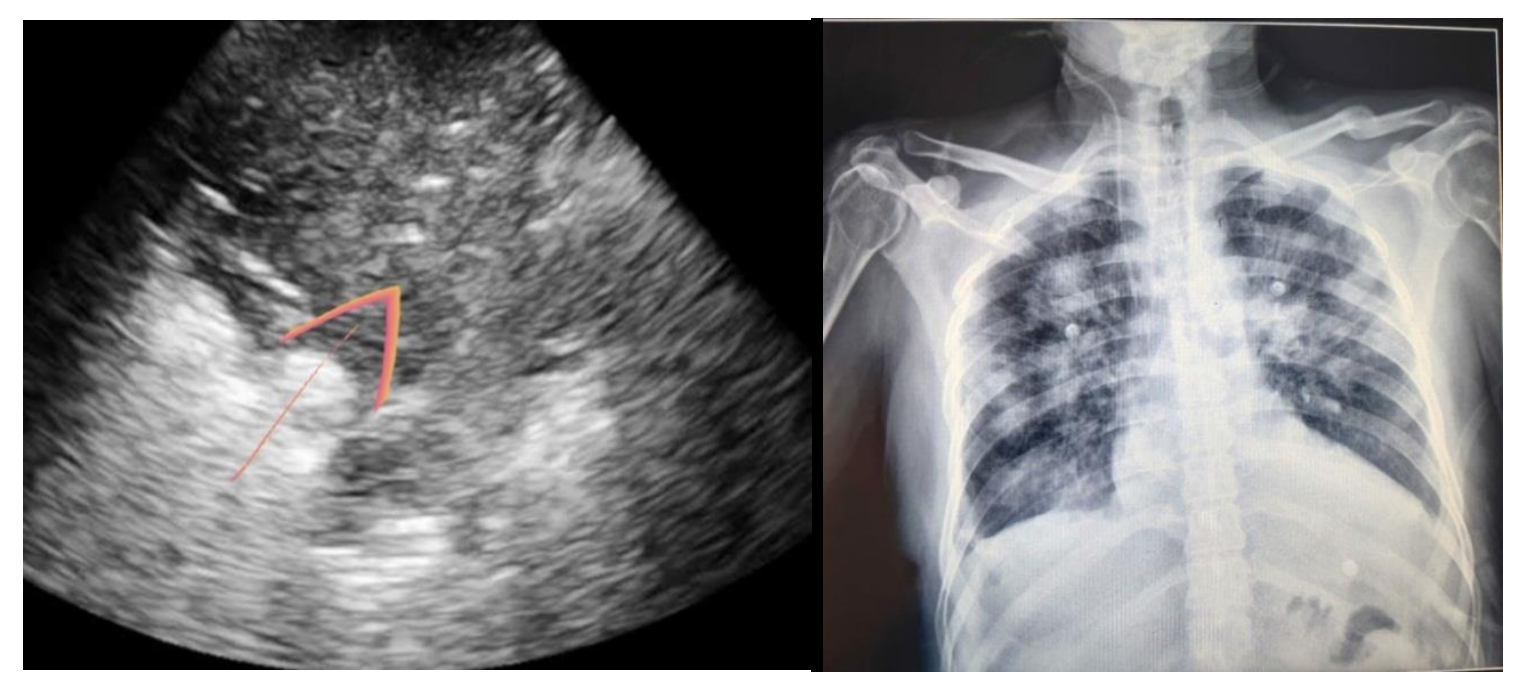

Figure (2 ): Picture of pneumonia in studied patients by LUS and CXR.
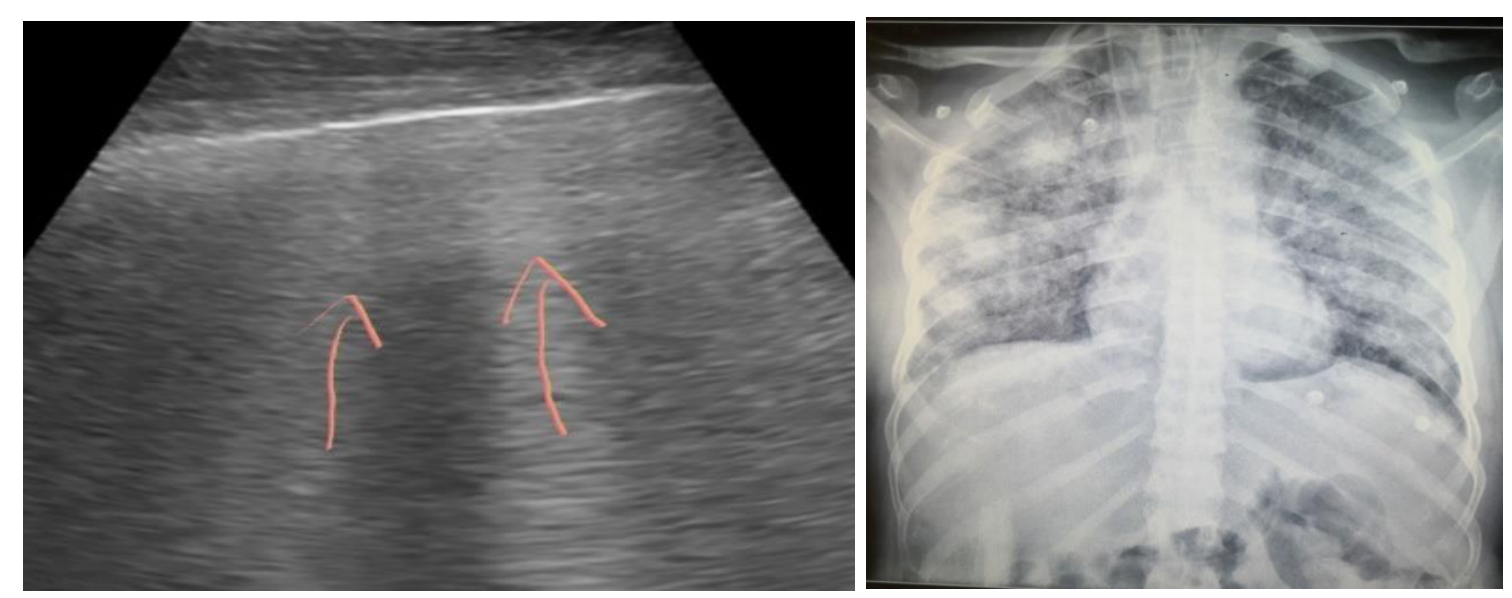

Figure (3 ): Picture of pulmonary edema in studied patients by LUS and CXR.
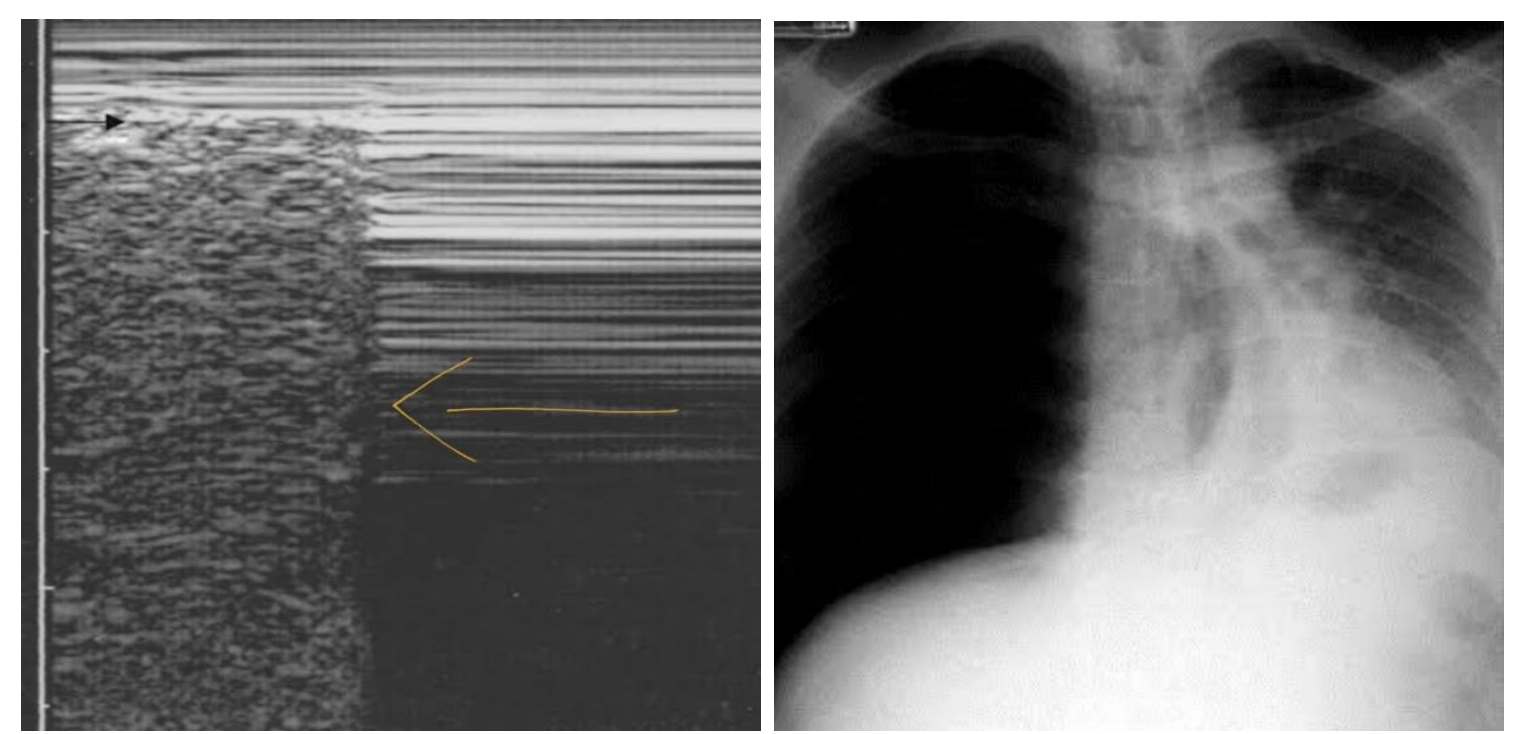

Figure (4): Picture of pneumothorax in our studied patients by LUS and CXR.

Our study showing that LUS has higher sensitivity and higher specificity than CXR and considered higher quality than CXR for the detection of pulmonary diseases and may be used as a replacement to thoracic $\mathrm{CT}$. 


\section{DISCUSSION}

Managing of critical ill patients need imaging methods, which are essential for diagnostic and therapeutic procedures. LUS has become a new method for detection of lung disease in mechanically ventilated cases. ICU physicians can use routinely LUS and can display exact information about lung condition with accurate diagnosis and therapeutic purpose. $^{5}$

The main principle of LUS is reduction of lung aeration with different diseases resulting in changing the lung surface and generating distinct profiles or patterns characteristic for each. The main advantages of ultrasound are that it is a relatively inexpensive, broadly available, rapid procedure, easily learned, and free from ionizing radiation or contrast. ${ }^{6}$

In this study sixty patients with acute dyspnea were admitted to ICU unit, they were 40 male and 20 female and their range of age (21-80) years old. They were $31(51.67 \%)$ smokers. dyspnea was the most common symptoms. More than half of the cases about $32(53.33 \%)$ cases receive oxygen by simple face mask.

CXR has limited diagnostic performance and efficacy due to poor quality $x$ ray films, expose the patients to radiation and need film cassette to placed posteriorly to the thorax. ${ }^{7}$

In this study CXR was abnormal in $48(80 \%)$ of cases, $36(60 \%)$ of them had homogenous opacity and $12(20 \%)$ had heterogenous opacity.

Costophrenic angle oblitrated in 15 (25\%) of cases. CXR diagnosis revealed 26 (43.33\%) of cases had consolidation, 16 (26.66\%) of cases had pleural effusion, 7 (11.66\%) had hyperinflated lung, 4 (6, $67 \%$ ) cases had pneumothorax and $2(3.33 \%)$ had pulmonary edema.

In this study we did ultrasound on the same day of addmition to ICU. Ultrasound showed lung sliding in $56(93.33 \%)$ of cases, diffuse B lines in $10(16$, $62 \%$ ) cases, focal B lines in $3(5 \%)$ cases, interrupted pleural line in $5(8,33 \%)$ cases, lung point were present in $4(6.67 \%)$ cases, consolidation were present in $24(40 \%)$ of cases, airbronchogram were present in $5(8.33 \%)$ of cases.

Consolidation was seen in ultrasound and is aspecial sign for pneumonia it is also present in lung atelectasis and ultrasound can differentiate pneumonia from atelectasis by presence of air bronchogram in atelectasis this also gives LUS an advantage over CXR. ${ }^{2}$

LUS revealed diagnosis 21 (35\%) of cases had pneumonia, $20(33.3 \%)$ of cases had pleural effusion, $4(6.66 \%)$ cases had pneumothorax,4 $(6.66 \%)$ cases had pulmonary edema,5 (8.33\%) cases had ARDS, and $14(23,33 \%)$ cases had COPD.

The incidence of pneumothorax among mechanically ventilated patients is high and is considered as one of the most serious complication of positive pressure breathing. Polytrauma cases especially with chest trauma may be present with Pneumothorax. However, unfortunately, it may be iatrogenic due to central venous catheter insertion, thoracocentesis and with positive pressure ventilation and all may necessitate chest tube. ${ }^{8}$

In current study LUS had sensitivity $100 \%$ and specificity $100 \%$ for diagnosis of pneumothorax versus $70 \%$ sensitivity and $81.8 \%$ specificity of CXR.As the small amount of pneumothorax not detected by CXR These result were in line with Felippe et al. ${ }^{9}$ who stated that ultrasound correctly diagnosed pneumothorax with percentage of sensitivity $79 \%$ and specificity $100 \%$. CXR had sensitivity $69 \%$ and specificity $100 \%$.

This finding is supported by result obtained by Dexheimer et al. ${ }^{9}$ who stated that pneumothorax can be excluded by ultrasound (sensitivity 100\%, specificity $83 \%$ ), as well as pneumothorax can be diagnosed as lung sliding not present, increase horizontal A lines and the loss of longitudinal B lines. ${ }^{9}$

Early and correct diagnosis helps to start early and effective treatment. Hence, we can solve this serious issue or at least decrease the morbidity related to it. In this study, we compared sensitivity and specificity of LUS with CXR. In our study, we concentrated only on lung consolidation as the diagnostic finding in LUS to detect pneumonia.

In our study LUS had sensitivity $93 \%$ and specificity $97 \%$ in diagnosis of pneumonia in correlation to CXR sensitivity $70 \%$ and specificity 95\% due to air bronchogram that seen by ultrasound in diagnosis of pneumonia and not seen in CXR. These results were in line with previous study reported by Nazerian et al. ${ }^{\mathbf{1 0}}$ that the sensitivity for diagnosis of pneumonia was higher by ultrasound than CXR ( $81 \%$ vs $64 \%$ respectively), while specificities were statistically similar between LUS and CXR (94\% and $90 \%$ respectively).

Also, our results that published by Cortellaro et al. ${ }^{11}$ that US showed a sensitivity of $99 \%$ and a specificity of $95 \%$ in correlation to CXR that $67 \%$ sensitivity and $85 \%$ specificity in diagnosis of pneumonia.

A common problem in ICU patients is Pleural effusion, inspite of decreased sensitivity and specificity of CXR,it was the only available method for diagnosis. So a new modality in diagnosis with high sensitivity and specificity is needed especially with positioning limitations for CXR. ${ }^{\mathbf{1 2}}$

In current study LUS had sensitivity $94 \%$ and specificity $96 \%$ in diagnosis of pleural effusion while CXR had sensitivity $70 \%$ and specificity $90 \%$ due to position limitation and need large amount at least 200c to be detected. These result are in line with Rocco et al. who reported that LUS had high sensitivity $94 \%$ 
and a high specificity of $99 \%$ while CXR had low sensitivity of $42 \%$ and low specificity of $97 \%$ in detection of pleural effusion. ${ }^{\mathbf{1 3}}$

Also, similar results reported by Sikora et al. ${ }^{14}$ that was done on 42 patients that had pleural effusion, found that CXR had low sensitivity of $65 \%$, low specificity of $81 \%$, in comparison to ultrasound that had high sensitivity of $100 \%$, high specificity of $100 \%$.

Pulmonary edema consider a life-threatening condition and the main cause for admission to ICU or may develop in the ICU that cause fluid accumulation in the lung parenchyma and air spaces impairing gas exchange which. Heart failure is a common cause of pulmonary edema, there is considerable uncertainty about the incidence of acute cardiogenic pulmonary edema. ${ }^{15}$

Pulmonary edema can be easily diagnosed by LUS. CXR can diagnose cardiogenic pulmonary edema only if there is butterfly opacity or vascular congestion however it is difficult to diagnose ARDS based on CXR alone. ${ }^{16}$

On the other hand cardiogenic pulmonary edema can be differentiate from non cardiogenic pulmonary edema by using LUS. ${ }^{17}$

In our study LUS detected cardiogenic pulmonary edema in $3(5 \%)$ cases and ARDS in 5 $(8.33 \%)$ cases. Cardiogenic pulmonary edema can be differentiate from non cardiogenic pulmonary edema by using LUS by thickening and irregularity of pleural line with presence of small subpleural consolidation to rule out cardiogenic pulmonary edema. ${ }^{18}$

In current study diagnosis of pulmonary edema by LUS had sensitivity $93 \%$ and specificity $93 \%$ in comparison to chest $\mathrm{X}$ ray which had sensitivity $36 \%$ and specificity $90 \%$ as the CXR not able to differentiate between two types of pulmonary edema (cardiogenic and non cardiogenic). These results are in line with those done by Lichtenstein and Mezière ${ }^{19}$ who performed that the LUS had sensitivity of $97 \%$ and specificity of $95 \%$ in diagnosis of pulmonary edema and CXR has sensitivity $40 \%$ and specificity $91 \%$.

\section{CONCLUSIONS}

Ultrasound is abed side tool useful for rapid and early diagnosis of acute dyspnea in critical ill patients. LUS has higher sensitivity and higher specificity of diagnosis of chest diseases as pneumonia, pleural diseases, pneumothorax and interstitial lung diseases in comparison to CXR.

Funding: This research did not receive any specific grant from funding agencies in the public, commercial, or not-for-profit sectors.

Acknowledgment: Author express your respectful thanks and profound gratitude to Dr. Ragia $\mathrm{H}$.
Hashem for her keen guidance, kind supervision, valuable advice and continuous encouragement, which made possible the completion of this work. Also express deepest gratitude and thanks to Dr. Soad S. Elgaby, for her kind care, continuous supervision, valuable instructions, constant help and great assistance throughout this work.also thankful to Dr.Eman S. Sobh, for her great help, active participation and guidance and introduce deep respect and thanks to Dr.Gehan A. Eldesoky, for her kindness, supervision and cooperation in this work.

Conflict of interest: None of the authors has conflicts of interest to declare.

Ethical approval statement: the study protocol was approved by the Institutional Review Board of Faculty of Medicine for Girls (AFMG-IRB) before patient enrollment in the study. Informed written consents obtained from patients themselves or from their relatives.

Author Contribution Statement: Ragia H. Hashem, Soad S. Elgaby, Eman S. Sobh, Gehan A. Eldesoky shared developing the research idea, designed the experiments, supervised the experiments performance, executed data analysis, wrote and revised the manuscript. Nourhan M. Abd El-Fattah performed the experiments, collected the data, carried out the graphical and statistical analysis and wrote the manuscript. The authors declare that all data were generated in-house and that no paper mill was used.

List of Abbreviations: intensive care units (ICU); Chest x-ray (CXR) ; lung ultrasound (LUS) ; Statistical Package for Social Science (SPSS); intra-class correlation coefficients (ICC); (CPAP) continuous positive air way pressure; Acute respiratory distress syndrome (ARDS).

\section{REFERENCES}

1. Brogi E, Bignami E, Sidoti A, Shawar M, et al. Could the use of bedside lung ultrasound reduce the number of chest $\mathrm{x}$-rays in the intensive care unit?. Cardiovascular ultrasound. $2017 ; 15(1): 1-5$.

2. Wang Y., Gargani L., Barskova T. Usefulness of lung ultrasound B-lines in connective tissue disease-associated interstitial lung disease: a literature review. Arthritis Res Ther. 2017; 19:206.

3. Kruisselbrink R, Chan V, Cibinel GA, Abrahamson S, Goffi A. I-AIM (indication, acquisition, interpretation, medical decision-making) framework for point of care lung ultrasound. Anesthesiology. 2017; 127(3):568-82. 
4. Rosner B. Fundamentals of Biostatistics. $7^{\text {th }}$ ed. Boston, MA: Brooks/Cole; 2018.

5. Barbry T, Bouhemad B, Leleu K, de Castro V, Remerand F, Rouby JJ. Transthoracic ultrasound approach of thoracic aorta in critically ill patients with lung consolidation. J Crit Care 2006; 21: 203208.

6. Alzahran SA, Al-Salamah MA, Al-Madani WH, and ElBarbary MA:Systematic review and meta-analysis forthe use of ultrasound versus radiology indiagnosing of pneumonia. Crit Ultras j.2017; 9: 1-11.

7. Winkler M.H., Touw H.R., van de Ven P.M. Diagnostic accuracy of chest radiograph, and when concomitantly studied lung ultrasound, in critically ill patients with respiratory symptoms: a systematic review and meta-analysis. Crit Care Med. 2018; 46:e707-e714.

8. Aziz SG, Patel BB, Ie SR, et al. The lung point sign, not pathognomonic of a pneumothorax. Ultrasound Quarterly 2016; 32:277-9.

9. Felipe, Dexheimer,Silva A, de Campos FP. "Neurothorax" complicating a misplaced nasogastric feeding tube in a severely ill patient. Autopsy \& case reports. 2012; 2(1):19.

10. Nazerian P, Volpicelli G, Vanni S, Gigli C, Betti L, Bartolucci M, et al. Accuracy of lung ultrasound for the diagnosis of consolidations when compared to chest computed tomography. The American journal of emergency medicine. 2015; 33(5):620-5.

11. Cortellaro F, Colombo S, Coen D, Duca PG. Lung ultrasound is an accurate diagnostic tool for the diagnosis of pneumonia in the emergency department. Emergency medicine journal. 2012; 29(1):19-23.

12. Maslove D, Chen BTM, Wang H, and Kuschner WG. The diagnosis and management of pleural effusions in the ICU. Inten Care Med. 2011; 28: 24-36.
13. Rocco M, Carbone I, Morelli A, et al. Diagnostic accuracy of bedside ultrasonography in the ICU: feasibility of detecting pulmonary effusion and lung contusion in patients on respiratory support after severe blunt thoracic trauma. Acta anaesthesiologica scandinavica. 2008; 52(6):776-84.

14. Sikora K, Perera P, Mailhot T, Mandavia D. Ultrasound for the detection of pleural effusions and guidance of the thoracentesis procedure. International Scholarly Research Notices. 2012; 2012.

15. Bourcier J-E, Paquet J, Seinger M, Gallard E, Redonnet J-P, Cheddadi F, et al. Performance comparison of lung ultrasound and chest X-ray for the diagnosis of pneumonia in the ED. Am J Emerg Med. 2014; 32(2): 115-118.

16. Copetti R, Soldati G, Copetti P. Chest sonography: A useful tool to differentiate acute cardiogenic pulmonary edema from acute respiratory distress syndrome. Cardiovasc Ultrasound 2018; 6:16.

17. Brogi E, Bignami E, Sidoti A, Shawar M, et al. Could the use of bedside lung ultrasound reduce the number of chest $\mathrm{x}$-rays in the intensive care unit? Cardiovascular ultrasound. 2017; 15(1):1-5.

18. Zhao Z, Jiang L, Xi X, Jiang Q, Zhu B, Wang $M$, et al. Prognostic value of extravascular lung water assessed with lung ultrasound score by chest sonography in patients with acute respiratory distress syndrome. BMC Pulm Med. 2015; 15:98.

19. Lichtenstein and Meziere. Relevance of lung ultrasound in the diagnosis of acute respiratory failure*: the BLUE protocol. Chest 2015; 134(1):117-25.

20. Lichtenstein DA. Lung ultrasound in the critically ill. Ann Intensive Care 2014:4(1):1. 